\title{
TILINGS OF CONVEX SETS BY MUTUALLY INCONGRUENT EQUILATERAL TRIANGLES CONTAIN ARBITRARILY SMALL TILES
}

\author{
CHRISTIAN RICHTER AND MELCHIOR WIRTH
}

\begin{abstract}
We show that every tiling of a convex set in the Euclidean plane $\mathbb{R}^{2}$ by equilateral triangles of mutually different sizes contains arbitrarily small tiles. The proof is purely elementary up to the discussion of one family of tilings of the full plane $\mathbb{R}^{2}$, which is based on a surprising connection to a random walk on a directed graph.
\end{abstract}

\section{INTRODUCTION AND MAIN RESULT}

Can the Euclidean plane $\mathbb{R}^{2}$ be tiled by equilateral triangles of pairwise different sizes? This problem, posed in [5, Exercise 2.4.10] and [4, Section C11], serves as the motivation for the present paper.

A tiling of a set $A \subseteq \mathbb{R}^{2}$ is a family $\mathcal{T}=\left\{T_{i}: i \in I\right\}$ of subsets $T_{i} \subseteq A$ with mutually disjoint interiors such that $A=\bigcup \mathcal{T}=\bigcup_{i \in I} T_{i}$. The elements $T_{i}$ of $\mathcal{T}$ are called tiles. We refer to [5] for a comprehensive survey on tilings in $\mathbb{R}^{2}$. Here we study tilings of convex sets $C$ by equilateral triangles $T_{i}$. The size of a triangle $T$ is measured by its diameter $\operatorname{diam}(T)=\sup _{x, y \in T}\|x-y\|$, i.e., by its longest side length. We are interested in the case when all tiles have pairwise different sizes. Such tilings by equilateral triangles are called perfect. This name is adopted from perfect tilings by squares [2].

Figure 1 illustrates a family of tilings of the full plane $\mathbb{R}^{2}$ by equilateral triangles depending on a parameter $\alpha \in\left(0, \frac{1}{2}\right]$. These tilings are not perfect since there are only three (if $\alpha<\frac{1}{2}$ ) or two (if $\alpha=\frac{1}{2}$ ) different sizes of tiles. The limit case $\alpha \downarrow 0$ (with triangles of size 0 being ignored) would give the hexagonal tiling by triangles of unit size.

The property of perfectness is rather restrictive. Tutte [11, p. 468] showed that there is no perfect tiling $\mathcal{T}$ of an equilateral triangle $T$ by finitely many equilateral triangles apart from the trivial one $\mathcal{T}=\{T\}$. This was generalized to tilings of bounded convex sets by Buchman [3] and by Tuza [12] (see also [6, Theorem 6]). Scherer 10 complemented this by a negative result on tilings of $\mathbb{R}^{2}$ and motivated the above introductory question.

Theorem 1. (a) There is no convex subset of $\mathbb{R}^{2}$ that possesses a perfect tiling by finitely many and at least two equilateral triangles [3, 12].

(b) A perfect tiling of the plane $\mathbb{R}^{2}$ by equilateral triangles does not contain a smallest tile [10].

We shall show the following result, thereby answering a question by Nandakumar [7, 4. Note 2].

Theorem 2. If a perfect tiling of a convex subset of the plane by equilateral triangles consists of at least two tiles, then it contains arbitrarily small tiles.

Date: March 22, 2022.

2000 Mathematics Subject Classification. 52C20 (primary), 05C81, 51M20, $60 J 10$ (secondary).

Key words and phrases. Perfect tiling, equilateral triangle, convex set, random walk, recurrent Markov chain. 


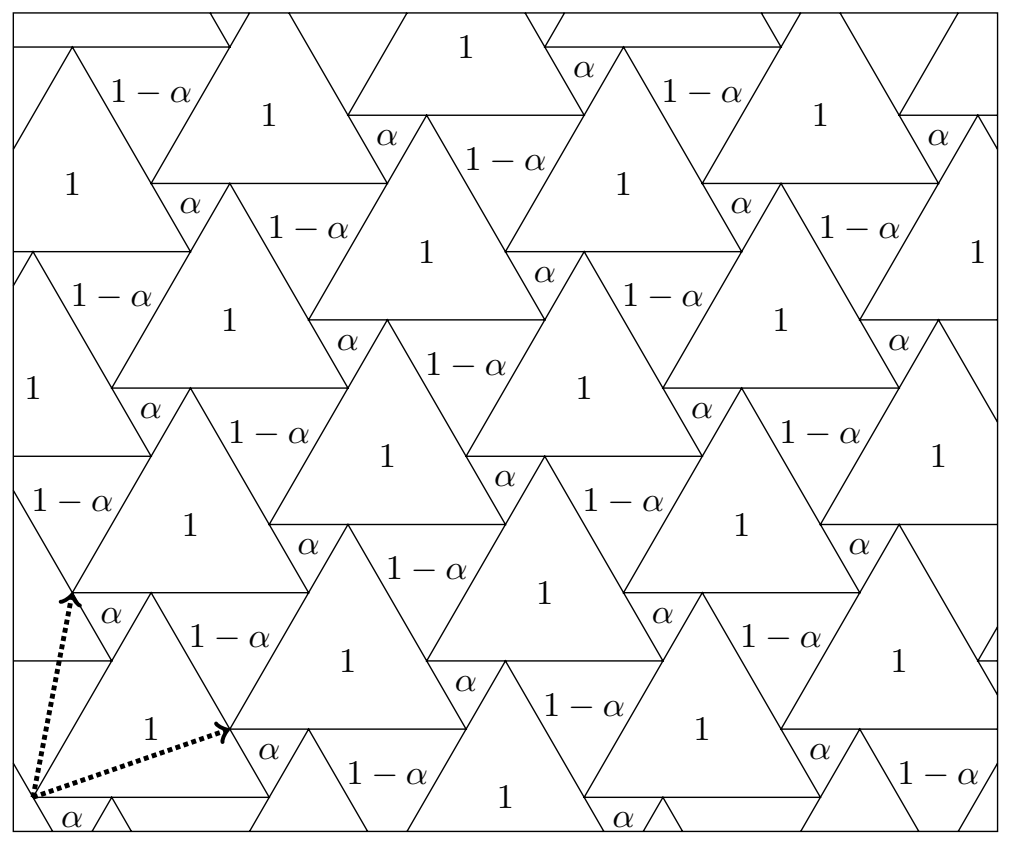

Figure 1. A family of tilings of $\mathbb{R}^{2}$ with parameter $0<\alpha \leq \frac{1}{2}$. The numbers stand for sizes of tiles. Arrows indicate periodicity.

Theorem 2 implies Theorem 1. The generalization of Theorem 1(b) is strict in so far as it excludes tilings of $\mathbb{R}^{2}$ such that the infimum of the sizes of all tiles is positive, but not attained as a minimum. The proof will be given in the following section. In parts it adopts an idea of Scherer [10], but does not make use of Theorem 1, This way it gives an alternative approach to Theorem 1(a) (see Subsections 2.2 and 2.3 below).

Often one requires a tiling to be locally finite, that is, every bounded subset of $\mathbb{R}^{2}$ meets at most finitely many tiles. For us local finiteness is not an a priory assumption. The general setting is much more flexible. In particular, it allows for perfect tilings by equilateral triangles of the plane $\mathbb{R}^{2}$ and of every open subset of $\mathbb{R}^{2}[8$, Corollary 2]. A convex polygon can be perfectly tiled if and only if all its inner angles are not smaller than $\frac{\pi}{3}$ [9, Corollary 7]. In fact, if a subset of $\mathbb{R}^{2}$ possesses a tiling by equilateral triangles, then it possesses a perfect tiling by equilateral triangles [9, Theorem 1].

Since all the tilings mentioned in the last paragraph exhibit accumulation phenomena, we formulate the remaining open question explicitly.

Problem 3. Does the plane $\mathbb{R}^{2}$ itself or any of its unbounded convex subsets possess a locally finite perfect tiling by equilateral triangles?

As byproducts of the proof of Theorem 2, we obtain a further necessary topological condition that is satisfied by every locally finite perfect tiling of an unbounded convex subset of $\mathbb{R}^{2}$ by equilateral triangles (see Corollary 13), and we characterize all locally finite tilings of (not necessarily unbounded) convex sets by (not necessarily incongruent) equilateral triangles that do not meet that condition (see Propositions 7 and 8 ).

Moreover, we shall prove a result in the spirit of two theorems from [6], which say that every locally finite tiling of the plane by arbitrary triangles with side lengths in the interval $[1,2)$ contains two triangles that share a side [6. Theorem 5] and that 


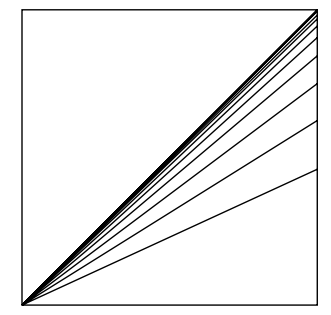

FiguRE 2. Tiling of a square by infinitely many triangles.

every finite tiling of a convex $k$-gon, $k \geq 4$, with arbitrary triangles contains two triangles that share a side [6, Theorem 6].

Theorem 4. Let $\mathcal{T}$ be a tiling of a convex subset of the plane by at least two equilateral triangles with a positive lower bound on the side lengths of all tiles. Then $\mathcal{T}$ contains two triangles that share a side or $\mathcal{T}$ is similar to a tiling of the type depicted in Figure 1 with suitable $\alpha \in\left(0, \frac{1}{2}\right]$.

Theorem 4 improves on a recent result on tilings of the complete plane $\mathbb{R}^{2}$ by Aduddell, Ascanio, Deaton and Mann [1, Theorem 1] in two ways. Firstly they assume that the tiling contains only tiles of finitely many different sizes, whereas we only impose a positive lower bound on the size of the tiles. Secondly their result requires an additional symmetry condition called equitransitivity, which we do not need at all.

\section{Proofs}

\subsection{Local finiteness.}

Lemma 5. If a tiling $\mathcal{T}$ by equilateral triangles is not locally finite, then it contains arbitrarily small tiles.

Proof. We use the notation $D\left(x_{0}, \varrho\right)=\left\{x \in \mathbb{R}^{2}:\left\|x-x_{0}\right\| \leq \varrho\right\}$ for the Euclidean disc with center $x_{0} \in \mathbb{R}^{2}$ and radius $\varrho>0 . \operatorname{Let} \operatorname{infdiam}(\mathcal{T})=\inf _{T \in \mathcal{T}} \operatorname{diam}(T)$.

Since $\mathcal{T}$ is not locally finite, there exist a disc $D\left(x_{0}, \varrho\right)$ and a sequence of distinct triangles $T_{1}, T_{2}, \ldots \in \mathcal{T}$ such that we can pick $x_{i} \in D\left(x_{0}, \varrho\right) \cap T_{i}, i=1,2, \ldots$ Let $T_{i}^{\prime} \subseteq T_{i}$ be obtained from $T_{i}$ by a dilation with center $x_{i}$ and factor $\frac{\operatorname{infdiam}(\mathcal{T})}{\operatorname{diam}\left(T_{i}\right)} \leq 1$. (Here degeneracy with factor 0 is not forbidden.) Then $\operatorname{diam}\left(T_{i}^{\prime}\right)=\operatorname{infdiam}(\mathcal{T})$ and $T_{i}^{\prime} \subseteq D\left(x_{0}, \varrho+\operatorname{infdiam}(\mathcal{T})\right)$ by the triangle inequality, because $x_{i} \in D\left(x_{0}, \varrho\right) \cap$ $T_{i}^{\prime}$. Consequently, $D\left(x_{0}, \varrho+\operatorname{infdiam}(\mathcal{T})\right)$ contains infinitely many non-overlapping equilateral triangles $T_{i}^{\prime}$ of size infdiam $(\mathcal{T})$. A volumetric argument yields our claim $\operatorname{infdiam}(\mathcal{T})=0$.

Lemma 5 on tilings by similar images of an equilateral triangle $T$ sounds trivial, but Figure 2 illustrates that tilings by affine images of $T$ may fail to be locally finite without containing arbitrarily small pieces.

2.2. E-configurations. Now we can restrict our consideration to locally finite tilings $\mathcal{T}$ of convex sets. Let $\operatorname{bd}(T)$ denote the boundary of a triangle $T$. The skeleton $\operatorname{skel}(\mathcal{T})=\bigcup_{T \in \mathcal{T}} \mathrm{bd}(T)$ of a tiling $\mathcal{T}$ by equilateral triangles is a union of segments of only three directions; say $v_{E}=(1,0)$ (east), $v_{N E}=\left(\frac{1}{2}, \frac{\sqrt{3}}{2}\right)$ (north east) and $v_{N W}=\left(-\frac{1}{2}, \frac{\sqrt{3}}{2}\right)$ (north west).

Next we follow an idea of Scherer [10. We denote the closed line segment between $x, y \in \mathbb{R}^{2}$ by $[x, y]$. For every $\varepsilon>0$ and every $0<\mu<1$, we call an isometric image of the set

$$
\left[(0,0), \lambda v_{E}\right] \cup\left[(0,0), \varepsilon v_{N W}\right] \cup\left[\mu \lambda v_{E}, \mu \lambda v_{E}+\varepsilon v_{N W}\right] \cup\left[\lambda v_{E}, \lambda v_{E}+\varepsilon v_{N W}\right]
$$


Case 2.
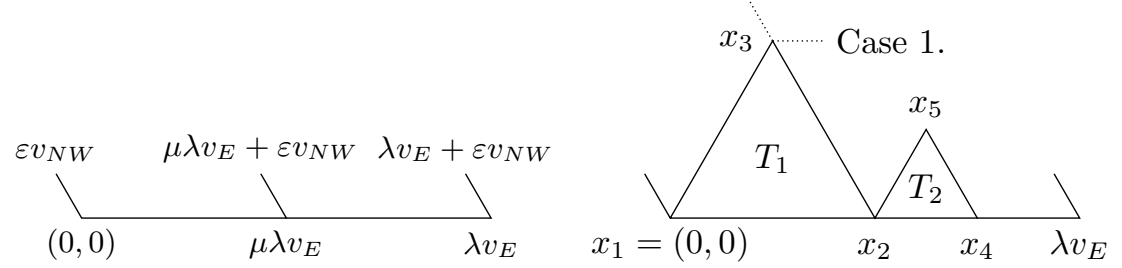

Figure 3. An E-configuration and the proof of Lemma 6
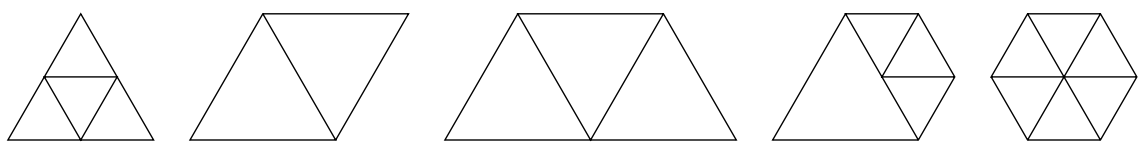

Figure 4. Finite tilings of bounded convex sets without $E$-configurations.

an E-configuration of length $\lambda>0$ (see the left-hand part of Figure 3). The respective isometric image of the segment $\left[(0,0), \lambda v_{E}\right]$ will be called the basis of the $E$-configuration. We say that $\mathcal{T}$ possesses an $E$-configuration of length $\lambda$ if $\operatorname{skel}(\mathcal{T})$ contains such a configuration as a subset.

Lemma 6 (cf. Scherer [10]). If a locally finite tiling $\mathcal{T}$ of a convex set $C \subseteq \mathbb{R}^{2}$ by equilateral triangles possesses an E-configuration and does not contain two triangles sharing a side, then $\mathcal{T}$ contains arbitrarily small tiles.

Proof. W.l.o.g. the tiling $\mathcal{T}$ possesses the $E$-configuration $E_{0}$ from (11). Since $\mathcal{T}$ covers the convex hull of $E_{0}$, there are triangles $T_{1}=\triangle x_{1} x_{2} x_{3}, T_{2}=\triangle x_{2} x_{4} x_{5} \in \mathcal{T}$ such that $x_{1}=(0,0), x_{2} \in\left[(0,0), \lambda v_{E}\right], x_{4} \in\left[x_{2}, \lambda v_{E}\right]$ and $x_{3}$ as well as $x_{5}$ have positive second coordinates (see the right-hand part of Figure 3).

If $\operatorname{skel}(\mathcal{T})$ contains a segment starting from $x_{3}$ in direction $v_{E}$ (Case 1 in Figure 3), then there is another $E$-configuration $E_{1}$ with basis $\left[x_{2}, x_{3}\right]$ since $\left[x_{2}, x_{2}\right]$ cannot be the side of another tile from $\mathcal{T}$. If not, then $\operatorname{skel}(\mathcal{T})$ must contain a segment starting from $x_{3}$ in direction $v_{N W}$ (Case 2 in Figure 3) and we obtain an $E$-configuration $E_{1}$ with basis $\left[x_{1}, x_{3}\right]$ by the same argument. In either case the length of $E_{1}$ is $\lambda_{1}=\operatorname{diam}\left(T_{1}\right)$. Since the length $\lambda$ of $E_{0}$ satisfies

$$
\lambda \geq \operatorname{diam}\left(T_{1}\right)+\operatorname{diam}\left(T_{2}\right) \geq \lambda_{1}+\operatorname{infdiam}(\mathcal{T}),
$$

the $E$-configuration $E_{1}$ has a length $\lambda_{1} \leq \lambda-\operatorname{infdiam}(\mathcal{T})$.

Iterating this procedure, we obtain $E$-configurations $E_{k}, k=1,2, \ldots$, of lengths $\lambda_{k} \leq \lambda-k \operatorname{infdiam}(\mathcal{T})$. Since all these lengths are positive, the claim $\operatorname{infdiam}(\mathcal{T})=0$ follows.

\subsection{Tilings of bounded convex sets without E-configurations.}

Proposition 7. Let $\mathcal{T}$ be a finite tiling of a bounded convex set $C \subseteq \mathbb{R}^{2}$ by at least two equilateral triangles and assume that $\mathcal{T}$ does not contain an E-configuration. Then $\mathcal{T}$ is the image of one of the five tilings of polygons from Figure 4 under a similarity transformation.

Proof. Here $C$ is a convex polygon with inner angles of sizes $\frac{\pi}{3}$ or $\frac{2 \pi}{3}$. Hence $C$ can be a triangle (three inner angles of $\frac{\pi}{3}$ ), a quadrilateral (two angles of $\frac{\pi}{3}$ and two of $\frac{2 \pi}{3}$ ) with parallelogram and trapezoid as subcases, a pentagon (one angle of $\frac{\pi}{3}$ and four of $\frac{2 \pi}{3}$ ) or a hexagon (six angles of $\frac{2 \pi}{3}$ ).

Since $\mathcal{T}$ does not contain an $E$-configuration, the sides of $C$ satisfy the following. 
(i) If at least one endpoint of a side $S$ of $C$ corresponds to an inner angle of $\frac{2 \pi}{3}$, then $S$ is a side of only one triangle of $\mathcal{T}$.

(ii) If a side $S$ of $C$ connects two vertices with inner angles of $\frac{\pi}{3}$, then there are at most two triangles in $\mathcal{T}$ each having one side in $S$.

Discussing the five cases for $C$ mentioned above under the restrictions (i) and (ii) gives the five tilings in Figure 4. (The trivial tiling $\mathcal{T}=\{C\}$ for the case when $C$ is a triangle is excluded by the assumption of Proposition 7).

Note that Lemma [6 and Proposition 7 give a new elementary approach to Theorem 1(a).

\subsection{Tilings of unbounded convex sets without E-configurations.}

Proposition 8. Let $\mathcal{T}$ be a locally finite tiling of an unbounded convex set $C \subseteq \mathbb{R}^{2}$ by equilateral triangles and suppose that $\mathcal{T}$ does not contain an E-configuration. Then $C=\mathbb{R}^{2}$ and $\mathcal{T}$ is the image of one of the tilings illustrated in Figure 1 under a similarity transformation.

The proof of Proposition 8 is based on two lemmas. The first one is elementary and concerns the topology of $\mathcal{T}$. The second one is given in the following subsection. We call two tilings $\mathcal{T}_{1}$ and $\mathcal{T}_{2}$ topologically equivalent (or having the same topology) if there is a bicontinuous bijection $\Phi: \bigcup \mathcal{T}_{1} \rightarrow \bigcup \mathcal{T}_{2}$ such that $\mathcal{T}_{2}=\left\{\Phi(T): T \in \mathcal{T}_{1}\right\}$ (cf. [5, p. 167]).

Lemma 9. Let $\mathcal{T}$ be a locally finite tiling of an unbounded convex set $C \subseteq \mathbb{R}^{2}$ by equilateral triangles and suppose that $\mathcal{T}$ does not contain an $E$-configuration. Then $C=\mathbb{R}^{2}$ and $\mathcal{T}$ contains a tiling $\mathcal{T}_{0} \subseteq \mathcal{T}$ that has the same topology as the one from Figure [1.

Proof. (A). Determination of $C$. Suppose that $C \neq \mathbb{R}^{2}$. Since $\mathcal{T}$ is locally finite and $C$ is unbounded, the boundary of $C$ contains a line or a half-line. There are infinitely many $E$-configurations having their bases in that line or half-line. So this case cannot appear, and we have $C=\mathbb{R}^{2}$.

(B). Topology of $\mathcal{T}$ in the neighbourhood of a maximal segment from $\operatorname{skel}(\mathcal{T})$. Let $S$ be a line segment in $\operatorname{skel}(\mathcal{T})$ that is maximal under inclusion. By the argument of (A), $S$ is bounded. W.l.o.g., $S=\left[x_{W}, x_{E}\right]$ is parallel to $v_{E}$ (see Subsection 2.2) and $x_{W}$ and $x_{E}$ are the west and east endpoints of $S$, respectively. Because of maximality of $S$, the endpoints $x_{W}, x_{E}$ are relatively inner points of two maximal segments $S_{W}, S_{E} \subseteq \operatorname{skel}(\mathcal{T})$, and they are in the relative interiors of sides of triangles $T_{W}, T_{E} \in \mathcal{T}$, respectively (see the left-hand part of Figure 5 a grey half-disc indicates that this region belongs to one single tile).

There are uniquely determined tiles $T_{N, 1}, \ldots, T_{N, n_{N}} \in \mathcal{T}$ and $T_{S, 1}, \ldots, T_{S, n_{S}} \in \mathcal{T}$ such that $S$ is the union of the south sides of $T_{N, 1}, \ldots, T_{N, n_{N}}$ as well as of the north sides of $T_{S, 1}, \ldots, T_{S, n_{S}}$. We can assume that

$$
n_{N} \leq n_{S} .
$$

Case 1: $n_{S} \geq 2$. First note that $n_{S} \leq 2$, since otherwise there would be an $E$-configuration with basis $S \cap\left(T_{S, 1} \cup T_{S, 2}\right)$. So we have $n_{S}=2$. Next note that $S_{W}$ must be parallel to $v_{N W}$ and $S_{E}$ parallel to $v_{N E}$, because otherwise we would again have an $E$-configuration with basis $S \cap\left(T_{S, 1} \cup T_{S, 2}\right)$. Then $n_{N}=1$, since $n_{N} \geq 2$ would give an $E$-configuration with basis $S \cap\left(T_{N, 1} \cup T_{N, 2}\right)$. So $n_{N}=1$, $n_{S}=2$, and the resulting topology is displayed in Figure 6 .

Case 2: $n_{S}=1$. Then $n_{N}=n_{S}=1$ and $S$ is a joint edge of $T_{N, 1}$ and $T_{S, 1}$. W.l.o.g., $S_{E}$ contains a side of $T_{N, 1}$ (see the right-hand part of Figure 5). The maximal segment $S_{E}$ contains sides of at least two triangles of $\mathcal{T}$, both being on the same side of $S_{E}$ as $S$. Hence $S_{E}$ is in the situation of Case 1 and the topology in its neighbourhood is as in Figure 6. In particular, $S_{E}$ is a side of $T_{E}$ and $S_{E}$ is 

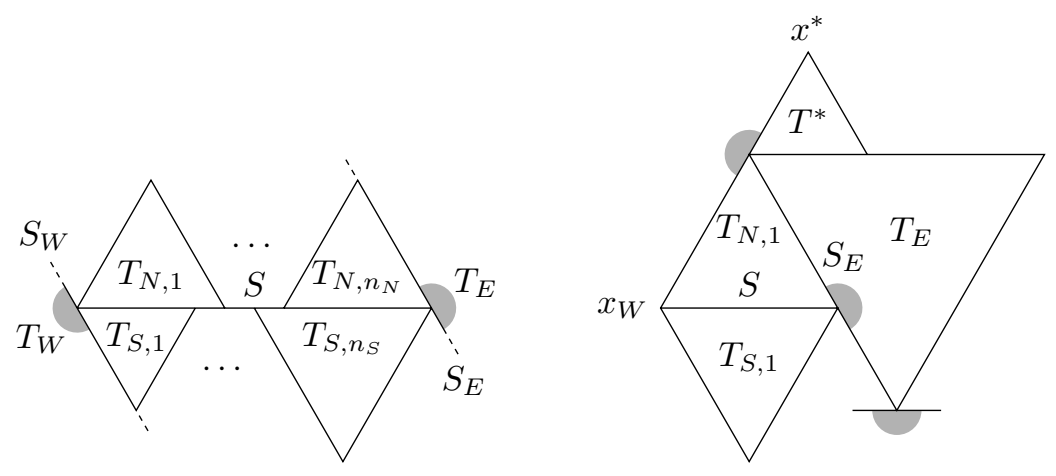

FiguRE 5. Notations in the neighbourhood of a maximal segment $S \subseteq \operatorname{skel}(\mathcal{T})$ and contradiction in Case 2 .

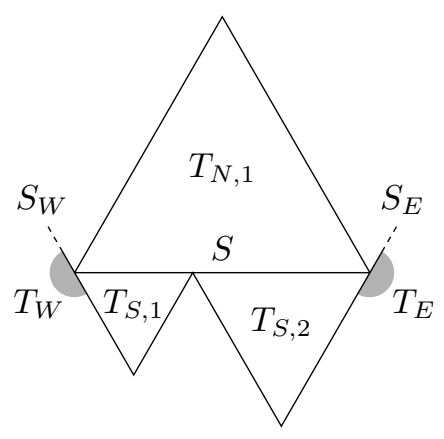

FIgURE 6 . Unique topology close to a maximal segment $S \subseteq \operatorname{skel}(\mathcal{T})$.

bounded at its north west endpoint by a segment in direction $v_{N E}$ and at its south east endpoint by a segment in direction $v_{E}$. Let $T^{*} \in \mathcal{T}$ be the tile whose south west vertex coincides with the north vertex of $T_{N, 1}$, and let $x^{*}$ be the north vertex of $T^{*}$. We obtain an $E$-configuration with basis $\left[x_{W}, x^{*}\right]$. This contradiction shows that Case 2 does not appear.

We have shown that the topology in a neighbourhood of every maximal line segment in $\operatorname{skel}(\mathcal{T})$ is as illustrated in Figure 6.

(C). Construction of $\mathcal{T}_{0}$. We shall identify triangles $T_{i, j}, L_{i, j}, R_{i, j} \in \mathcal{T}$ for $(i, j) \in \mathbb{Z}_{\text {even }}^{2}=\left\{(i, j) \in \mathbb{Z}^{2}: i+j\right.$ is even $\}$. The triangles $T_{i, j}$ are the equivalents of the triangles of size 1 from Figure 1, so that $T_{i, j+2}, T_{i-1, j-1}$ and $T_{i+1, j-1}$ meet the north, south west and south east vertex of $T_{i, j}$. The triangles $L_{i, j}$ and $R_{i, j}$ are the equivalents of the triangles of sizes $\alpha$ and $1-\alpha$, respectively, which touch $T_{i, j}$ along their north sides. The construction is illustrated in Figure 7 Pieces of information obtained in Steps 0, 1 and 2 are marked by (1), (1) and (2), respectively.

Step 0. We pick a maximal segment $S \subseteq \operatorname{skel}(\mathcal{T})$. By part (B), we can assume that the situation close to $S$ is as in Figure 6. We define $T_{0,0}=T_{N, 1}, L_{0,0}=T_{S, 1}$ and $R_{0,0}=T_{S, 2}$.

Step 1. The north east side $S_{1}$ of $T_{0,0}$ is parallel to $v_{N W}$ and is bounded at its south east endpoint by a segment in direction $v_{N E}$. By (B), this implies that $S_{1}$ is a maximal line segment of $\operatorname{skel}(\mathcal{T}), S_{1}$ is bounded at its north west endpoint by a segment in direction $v_{E}$, and $S_{1}$ is the union of two sides of triangles $L_{1,1}, R_{0,2} \in \mathcal{T}$ opposite to $T_{0,0}$ (where $R_{0,2}$ is in the north west of $L_{1,1}$ ). Similarly, the north west side of $T_{0,0}$ is the union of two sides of triangles $R_{-1,1}, L_{0,2} \in \mathcal{T}$ opposite to $T_{0,0}$. 


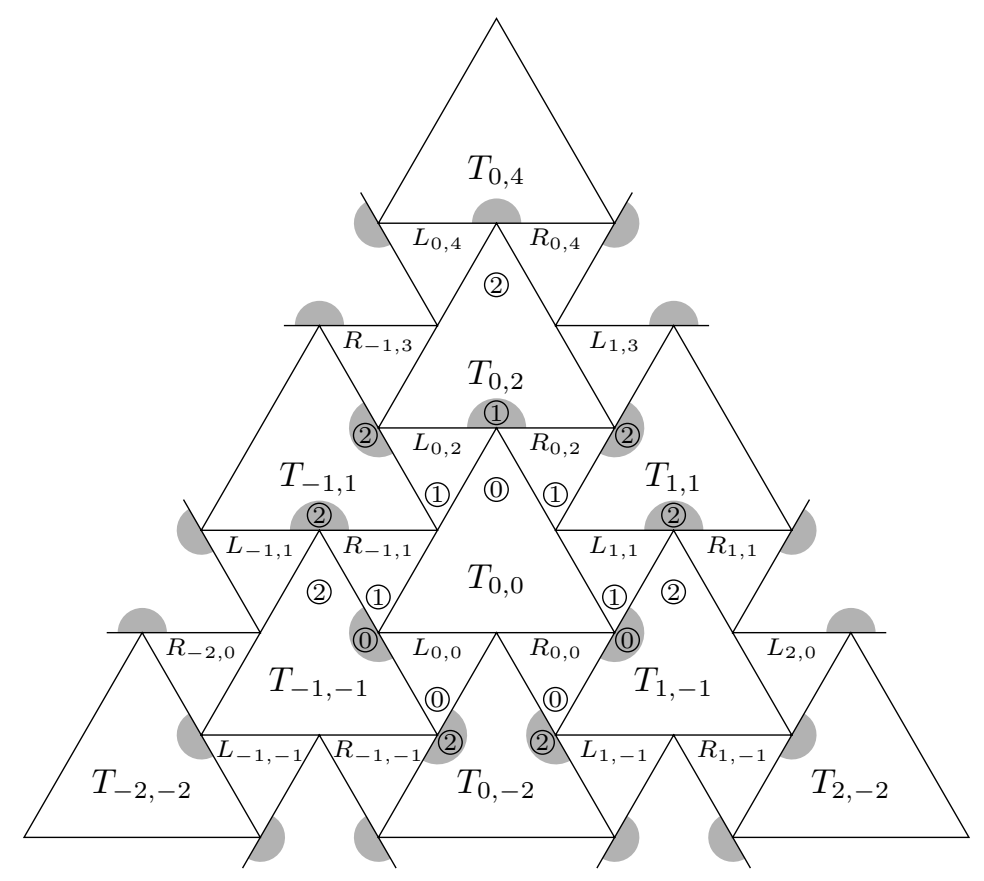

Figure 7. Construction of $\mathcal{T}_{0}$.

Step 2. Now consider the segment $S_{2}$ formed by the collinear sides of $L_{0,2}$ and $R_{0,2}$. By (B), $S_{2}$ is a maximal segment in $\operatorname{skel}(\mathcal{T}), S_{2}$ is bounded at its west endpoint by a segment in direction $v_{N W}$ and at its east endpoint by a segment in direction $v_{N E}$, and $S_{2}$ is a side of some $T_{0,2} \in \mathcal{T}$ opposite to $L_{0,2}$ and $R_{0,2}$. Similar considerations apply to segments formed by collinear sides of $R_{-1,1}$ and $L_{0,0}$ as well as of $R_{0,0}$ and $L_{1,1}$. In particular, we obtain tiles $T_{-1,-1}$ opposite to $R_{-1,1}$ and $L_{0,0}$ and $T_{1,-1}$ opposite to $R_{0,0}$ and $L_{1,1}$.

Step 3. Now we know that one side of each of $T_{0,2}, T_{-1,-1}$ and $T_{1,-1}$ is in the same topological situation as was the initial segment $S$ with respect to $T_{0,0}$ after Step 0. We repeat Steps 1 and 2 with these three maximal segments and arrive at the topological structure displayed in Figure 7.

Iterating this procedure, we get the desired tiling $\mathcal{T}_{0} \subseteq \mathcal{T}$.

2.5. The remaining topological type and a random walk. We associate a directed graph $\Gamma=\left(\mathbb{Z}_{\text {even }}^{2}, E\right)$ with vertex set $\mathbb{Z}_{\text {even }}^{2}$ to the triangles $T_{i, j} \in \mathcal{T}_{0}$ constructed above. Two vertices $(i, j),(k, l) \in \mathbb{Z}_{\text {even }}^{2}$ are connected by an edge, $[(i, j),(k, l)] \in E$, if and only if some vertex of $T_{i, j}$ belongs to $\operatorname{bd}\left(T_{k, l}\right)$. That is,

$$
[(i, j),(k, l)] \in E \quad \Longleftrightarrow \quad(k, l) \in\{(i-1, j-1),(i+1, j-1),(i, j+2)\}
$$

(see Figure 8). We consider the random walk on $\Gamma$ with transition probability $p((i, j),(k, l))=\frac{1}{3}$ for $[(i, j),(k, l)] \in E$ (and in turn $p((i, j),(k, l))=0$ for $[(i, j),(k, l)] \notin E)$. This is described by the Markov chain $\left(\mathbb{Z}_{\text {even }}^{2}, P\right)$ with state space $\mathbb{Z}_{\text {even }}^{2}$ and transition matrix $P=(p((i, j),(k, l)))_{(i, j),(k, l) \in \mathbb{Z}_{\text {even }}^{2}}$ (cf. [13, pp. $3-11])$.

Note that, for any two states $(i, j),(k, l) \in \mathbb{Z}_{\text {even }}^{2}$, there is a path from $(i, j)$ to $(k, l)$ in $\Gamma$. Equivalently, the probability to reach $(k, l)$ within finitely many steps when starting at $(i, j)$ is positive. Such Markov chains are called irreducible [13, p. 28]. 


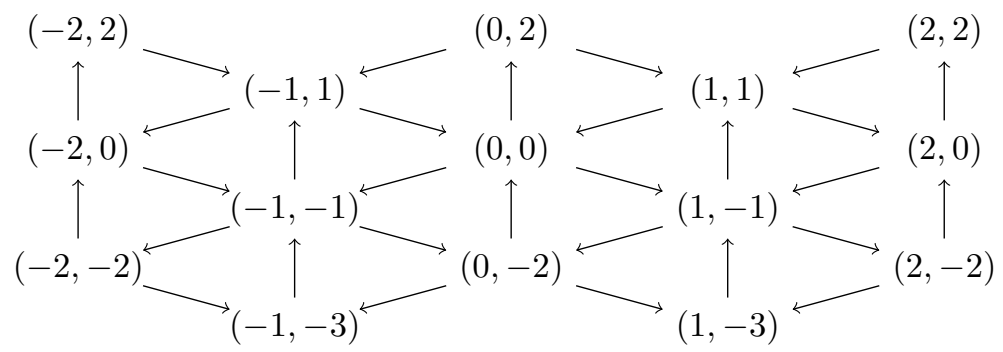

Figure 8. The directed graph $\Gamma=\left(\mathbb{Z}_{\text {even }}^{2}, E\right)$.

The probability $p^{(n)}((i, j),(k, l))$ of reaching the state $(k, l)$ from the state $(i, j)$ in exactly $n$ steps, $n \in\{0,1, \ldots\}$, is the respective entry of the $n$th power $P^{n}$ of $P$ [13. pp. 12-13, Lemma 1.21(a)]. That is,

$$
p^{(n)}((i, j),(k, l))=\sum_{\pi} p\left((i, j),\left(i_{1}, j_{1}\right)\right) p\left(\left(i_{1}, j_{1}\right),\left(i_{2}, j_{2}\right)\right) \ldots p\left(\left(i_{n-1}, j_{n-1}\right),(k, l)\right)
$$

with summation over all paths $\pi=\left[(i, j),\left(i_{1}, j_{1}\right),\left(i_{2}, j_{2}\right), \ldots,\left(i_{n-1}, j_{n-1}\right),(k, l)\right]$ of length $n$ in $\Gamma$ (i.e., $\left.\left[(i, j),\left(i_{1}, j_{1}\right)\right],\left[\left(i_{1}, j_{1}\right),\left(i_{2}, j_{2}\right)\right], \ldots,\left[\left(i_{n-1}, j_{n-1}\right),(k, l)\right] \in E\right)$.

In particular, the probability $p^{(n)}((i, j),(i, j))$ of returning from $(i, j)$ to $(i, j)$ in exactly $n$ steps is

$$
p^{(n)}((i, j),(i, j))=\sum_{\pi}\left(\frac{1}{3}\right)^{n}
$$

with summation over all cycles $\pi$ of length $n$ that start at $(i, j)$.

Recall that every edge in $E$ is of the form $[(k, l),(k, l)+(-1,-1)],[(k, l),(k, l)+$ $(1,-1)]$ or $[(k, l),(k, l)+(0,2)]$. Since the vectors $(-1,-1),(1,-1)$ and $(0,2)$ are pairwise linearly independent, every cycle must have length $n=3 m$ for some $m \in$ $\{0,1, \ldots\}$ and contain $m$ edges of each of these three types. Therefore the number of cycles of length $3 m$ starting at $(i, j)$ is $\frac{(3 m) !}{m ! m ! m !}$, which counts the possible consecutive orders of these three types of edges. Thus

$$
p^{(n)}((i, j),(i, j))= \begin{cases}\frac{(3 m) !}{3^{3 m} m ! m ! m !} & \text { if } n=3 m, m \in\{0,1, \ldots\}, \\ 0 & \text { otherwise. }\end{cases}
$$

The expected number of visits of the state $(i, j)$, provided the random walk starts at $(i, j)$, is

$$
G((i, j),(i, j))=\sum_{n=0}^{\infty} p^{(n)}((i, j),(i, j))=\sum_{m=0}^{\infty} \frac{(3 m) !}{3^{3 m}(m !)^{3}}
$$

[13. p. 15]. Using Stirling's estimates $\sqrt{2 \pi} m^{m+\frac{1}{2}} e^{-m} \leq m ! \leq e m^{m+\frac{1}{2}} e^{-m}$ for $m \in\{1,2, \ldots\}$, we obtain

$$
G((i, j),(i, j)) \geq \sum_{m=1}^{\infty} \frac{\sqrt{2 \pi}(3 m)^{3 m+\frac{1}{2}} e^{-3 m}}{3^{3 m}\left(e m^{m+\frac{1}{2}} e^{-m}\right)^{3}}=\frac{\sqrt{6 \pi}}{e^{3}} \sum_{m=1}^{\infty} \frac{1}{m}=\infty
$$

for all $(i, j) \in \mathbb{Z}_{\text {even }}^{2}$.

A state $(i, j)$ is called recurrent if $G((i, j),(i, j))=\infty$ [13, Theorem 3.4(a) and Definition 3.1], and a Markov chain is called recurrent if all its states are recurrent. An irreducible Markov chain is recurrent if and only if one of its states is recurrent [13, pp. $45-46,48]$.

Lemma 10. The Markov chain $\left(\mathbb{Z}_{\text {even }}^{2}, P\right)$ is recurrent. 
A function $f: \mathbb{Z}_{\text {even }}^{2} \rightarrow \mathbb{R}$ is called harmonic with respect to $\left(\mathbb{Z}_{\text {even }}^{2}, P\right)$ if it satisfies the mean value property $f(i, j)=\sum_{(k, l) \in \mathbb{Z}_{\text {even }}^{2}} p((i, j),(k, l)) f(k, l)$ for all $(i, j) \in \mathbb{Z}_{\text {even }}^{2}[13$, Definition 6.13]; i.e.,

$$
f(i, j)=\frac{1}{3}(f(i-1, j-1)+f(i+1, j-1)+f(i, j+2)) .
$$

By [13, Theorem 6.21], every non-negative harmonic function of a recurrent irreducible Markov chain is constant.

Corollary 11. Every non-negative harmonic function of $\left(\mathbb{Z}_{\text {even }}^{2}, P\right)$ is constant.

We come back to tilings by equilateral triangles.

Lemma 12. If a tiling $\mathcal{T}_{0}$ by equilateral triangles has the same topology as the one from Figure 1, then $\mathcal{T}_{0}$ is the image of a tiling of the family depicted in Figure 1 under a similarity transformation of the plane.

Proof. Adopting notation from the above construction of $\mathcal{T}_{0}$ (see Figure 7 ), we suppose that $\mathcal{T}_{0}$ consists of the triangles $T_{i, j}, L_{i, j}, R_{i, j}$ with $(i, j) \in \mathbb{Z}_{\text {even }}^{2}$ and that the three sides of $T_{i, j}$ split into the collinear sides of $L_{i, j}$ and $R_{i, j}$, of $L_{i+1, j+1}$ and $R_{i, j+2}$ and of $L_{i, j+2}$ and $R_{i-1, j+1}$, respectively. In particular,

$$
\begin{aligned}
\operatorname{diam}\left(T_{i, j}\right) & =\operatorname{diam}\left(L_{i, j}\right)+\operatorname{diam}\left(R_{i, j}\right) \\
& =\operatorname{diam}\left(L_{i+1, j+1}\right)+\operatorname{diam}\left(R_{i, j+2}\right) \\
& =\operatorname{diam}\left(L_{i, j+2}\right)+\operatorname{diam}\left(R_{i-1, j+1}\right) .
\end{aligned}
$$

Summing these three equations and then applying (4) to $T_{i-1, j-1}$, (5) to $T_{i+1, j-1}$ and (3) to $T_{i, j+2}$, we obtain

$$
\begin{aligned}
3 \operatorname{diam}\left(T_{i, j}\right)= & \left(\operatorname{diam}\left(L_{i, j}\right)+\operatorname{diam}\left(R_{i-1, j+1}\right)\right)+\left(\operatorname{diam}\left(L_{i+1, j+1}\right)+\operatorname{diam}\left(R_{i, j}\right)\right) \\
& +\left(\operatorname{diam}\left(L_{i, j+2}\right)+\operatorname{diam}\left(R_{i, j+2}\right)\right) \\
= & \operatorname{diam}\left(T_{i-1, j-1}\right)+\operatorname{diam}\left(T_{i+1, j-1}\right)+\operatorname{diam}\left(T_{i, j+2}\right) .
\end{aligned}
$$

Hence the function $f: \mathbb{Z}_{\text {even }}^{2} \rightarrow \mathbb{R}, f(i, j)=\operatorname{diam}\left(T_{i, j}\right)$, satisfies the harmonicity condition (2). As $f(i, j)=\operatorname{diam}\left(T_{i, j}\right)>0$ for all $(i, j) \in \mathbb{Z}_{\text {even }}^{2}$, Corollary 11 shows that $f$ is constant. Since we have to characterize $\mathcal{T}_{0}$ only up to similarity, we can assume that

$$
\operatorname{diam}\left(T_{i, j}\right)=1 \quad \text { for all } \quad(i, j) \in \mathbb{Z}_{\text {even }}^{2}
$$

Now

$$
\begin{aligned}
\operatorname{diam}\left(L_{i, j+2}\right) & \stackrel{\sqrt{5}}{=} \operatorname{diam}\left(T_{i, j}\right)-\operatorname{diam}\left(R_{i-1, j+1}\right) \\
& \stackrel{\sqrt{\underline{6}}}{=} \operatorname{diam}\left(T_{i, j}\right)-\left(\operatorname{diam}\left(T_{i-1, j-1}\right)-\operatorname{diam}\left(L_{i, j}\right)\right) \\
& \stackrel{\sqrt[6]{=}}{=} \operatorname{diam}\left(L_{i, j}\right)
\end{aligned}
$$

and

$$
\begin{aligned}
& \operatorname{diam}\left(L_{i+1, j+1}\right) \stackrel{\text { 点 }}{=} \operatorname{diam}\left(T_{i+1, j-1}\right)-\operatorname{diam}\left(R_{i, j}\right) \\
& \stackrel{\text { (3) }}{=} \operatorname{diam}\left(T_{i+1, j-1}\right)-\left(\operatorname{diam}\left(T_{i, j}\right)-\operatorname{diam}\left(L_{i, j}\right)\right) \\
& \text { (6) } \operatorname{diam}\left(L_{i, j}\right) \text {. }
\end{aligned}
$$

W.l.o.g., $\operatorname{diam}\left(L_{0,0}\right) \leq \operatorname{diam}\left(R_{0,0}\right)$. Then the parameter $\alpha=\operatorname{diam}\left(L_{0,0}\right)$ satisfies

$$
0<\alpha \leq \frac{1}{2}
$$


because $\operatorname{diam}\left(L_{0,0}\right)+\operatorname{diam}\left(R_{0,0}\right) \stackrel{(3)}{=} \operatorname{diam}\left(T_{0,0}\right) \stackrel{(6)}{=} 1$, and the periodicities (7) and (8) yield

$$
\operatorname{diam}\left(L_{i, j}\right)=\alpha \quad \text { for all } \quad(i, j) \in \mathbb{Z}_{\text {even }}^{2}
$$

Finally, (3), (6) and (10) imply

$$
\operatorname{diam}\left(R_{i, j}\right)=1-\alpha \quad \text { for all } \quad(i, j) \in \mathbb{Z}_{\text {even }}^{2}
$$

Claims (6), (10), (11) and (9) prove Lemma 12

Proof of Proposition 8. Lemmas 9 and 12 show that $C=\mathbb{R}^{2}$ and that $\mathcal{T}$ contains a tiling $\mathcal{T}_{0}$ that is a similar image of one illustrated in Figure 1, We obtain $\mathcal{T}=\mathcal{T}_{0}$, because $\bigcup \mathcal{T}_{0}=\mathbb{R}^{2}=\bigcup \mathcal{T}$.

\subsection{Conclusion.}

Proof of Theorem 2 Lemmas 5 and 6 discuss tilings that are not locally finite and locally finite tilings possessing $E$-configurations. Propositions 7 and 8 exclude locally finite tilings without $E$-configurations for bounded and for unbounded convex sets, respectively, by showing that they cannot be perfect.

Proof of Theorem 4. By Lemma 5, the tiling $\mathcal{T}$ is necessarily locally finite. Lemma 6 settles the case of tilings with $E$-configurations. Propositions 7 and 8 discuss tilings without $E$-configurations.

Finally, let us point out that Proposition 8 and the proof of Lemma 6 give rise to the following necessary condition in the context of Problem 3 .

Corollary 13. Every locally finite perfect tiling of an unbounded convex subset of the plane $\mathbb{R}^{2}$ by equilateral triangles possesses a sequence of E-configurations of strictly decreasing lengths.

\section{REFERENCES}

[1] R. Aduddell, M. Ascanio, A. Deaton, C. Mann: Unilateral and equitransitive tilings by equilateral triangles, Discrete Math. 340 (2017), 1669-1680.

[2] R.L. Brooks, C.A.B. Smith, A.H. Stone, W.T. Tutte: The dissection of rectangles into squares, Duke Math. J. 7 (1940), 312-340.

[3] E. Buchman: The impossibility of tiling a convex region with unequal equilateral triangles, Amer. Math. Monthly 88 (1981), 748-753.

[4] H.T. Croft, K.J. Falconer, R.K. Guy: Unsolved problems in geometry, Problem Books in Mathematics. Unsolved Problems in Intuitive Mathematics, II. Springer-Verlag, New York, 1991.

[5] B. Grünbaum, G.C. Shephard: Tilings and patterns, W.H. Freeman and Company, New York, 1987.

[6] A. Kupavskii, J. Pach, G. Tardos: Tilings with noncongruent triangles, arXiv:1711.04504, November 13, 2017.

[7] R. Nandakumar: Non-Congruent Tiling - an Ongoing Story, blog entry, http://nandacumar.blogspot.de/2016/06/non-congruent-tiling-ongoing-story.html June 14, 2016.

[8] C. Richter: Tiling by incongruent equilateral triangles without requiring local finiteness, Elem. Math. 67 (2012), 157-163.

[9] C. Richter: Tiling by incongruent equilateral triangles without requiring local finiteness, Part II, Elem. Math. (to appear).

[10] K. Scherer: The impossibility of a tesselation of the plane into equilateral triangles whose sidelengths are mutually different, one of them being minimal, Elem. Math. 38 (1983), 1-4.

[11] W.T. Tutte: The dissection of equilateral triangles into equilateral triangles, Proc. Cambridge Philos. Soc. 44 (1948), 463-482.

[12] Z. Tuza: Dissections into equilateral triangles, Elem. Math. 46 (1991), 153-158.

[13] W. Woess: Denumerable Markov chains. Generating functions, boundary theory, random walks on trees, EMS Textbooks in Mathematics, European Mathematical Society (EMS), Zürich, 2009. 
Institute of Mathematics, Friedrich Schiller University, D-07737 Jena, Germany

E-mail address: christian.richter@uni-jena.de

Institute of Mathematics, Friedrich Schiller University, D-07737 Jena, Germany

E-mail address: melchior.wirth@uni-jena.de 